\title{
Antiphospholipid Syndrome Complicated by Unilateral Pleural Effusion
}

\author{
Yasutaka Mitamura Masakazu Takahara Takamichi Ito Misa Nakano \\ Yoichi Moroi Masutaka Furue \\ Department of Dermatology, Graduate School of Medical Sciences, Kyushu University, \\ Fukuoka, Japan
}

\section{Key Words}

Antiphospholipid syndrome · Pleural effusion · Corticosteroids · Lupus-like disease

\begin{abstract}
Antiphospholipid syndrome (APS) with pleural effusion is extremely rare. A 75-year-old man was admitted to our hospital for spreading erythema on his trunk and extremities, as well as dyspnea. One year before admission, he had visited us with a 1-year history of erythema and purpura on his legs and occasional fever. Given the diagnosis of APS, we initiated a combination therapy of aspirin and warfarin, but the skin lesions had gradually worsened. A biopsy specimen revealed marked thrombosis in the dermal and subcutaneous small vessels. In addition, chest X-ray and computed tomography demonstrated a large pleural effusion in the left lung. He underwent repeated drainage of the pleural effusion but the effusion recurred. We added oral prednisolone $30 \mathrm{mg}$ daily to his prior anticoagulant therapy. The skin lesions and pleural effusion rapidly improved and disappeared without any complication. Corticosteroids might be a choice of treatment for intractable pleural effusion in APS patients.

(c) 2013 S. Karger AG, Basel
\end{abstract}

\section{Introduction}

Antiphospholipid syndrome (APS) is an autoimmune disease characterized by thrombosis and morbidity, specifically in pregnancy, due to antiphospholipid antibodies. About half of the cases of APS occur as a primary disorder, while the rest arise in association with other autoimmune diseases, such as systemic lupus erythematosus (SLE) [1]. Some diseases, such as pulmonary thrombosis and pulmonary hypertension, are known to be complicated

Yasutaka Mitamura

Department of Dermatology

Graduate School of Medical Sciences, Kyushu University

3-1-1 Maidashi, Higashiku, Fukuoka 812-8582 (Japan)

E-Mailmitamura@med.kyushu-u.ac.jp 
by APS [2]; however, APS with pleural effusion is extremely rare. Here, we present a case of APS complicated by unilateral pleural effusion that responded well to oral corticosteroid therapy.

\section{Case Presentation}

A 75-year-old Japanese man was admitted to our hospital for spreading erythema on his trunk and extremities, as well as dyspnea. One year prior to admission, he visited us with a 1-year history of erythema and purpura on his legs, accompanied by intermittent fever. Results of laboratory examinations for antiphospholipid antibodies, lupus anticoagulant (using the phospholipid neutralization test), and anticardiolipin antibody had been positive 12 weeks apart. In addition, he showed positive antinuclear antibody (1:80, homogeneous pattern), but was negative for anti-dsDNA antibody, anti-Sm antibody, anti-RNP antibody, anti-SS-A antibody, anti-SS-B antibody, antitopoisomerase I antibody, and anticentromere antibody. MPO-ANCA, PR3-ANCA, and cryoglobulin were negative. Given the diagnosis of APS, we initiated combination therapy with aspirin (100 mg daily) and warfarin (target international normalized ratio, 2.0-3.0), but the skin lesions continued to gradually worsen. Violaceous erythema, purpura, and pigmentation were widely noted on his trunk and extremities (fig. 1); they were associated with low platelets $(93,000 / \mu \mathrm{l})$ and elevated partial thromboplastin time (48.4 s). A biopsy specimen revealed marked thrombosis in the dermal and subcutaneous small vessels (fig. 2). There were interface changes of the dermoepidermal junction and mild inflammatory infiltrates in the perivascular area of the dermis, but mucin deposition and thickening of the basal layer of the epidermis were not apparent. In addition, a chest X-ray and computed tomography demonstrated a large pleural effusion in the left lung (fig. 3), without evidence of large vessel thrombus. Electrocardiogram and echocardiogram were normal. Despite serial thoracenteses, effusion recurred. Thoracoscopy and parietal pleura biopsy showed only marked lymphocytic infiltration without evidence of malignancy. An analysis of the pleural fluid also indicated a benign exudative effusion. Bacterial and fungal cultures, as well as cytology analyses for malignant cells, were all negative. After excluding infectious diseases, malignancies, pulmonary thrombosis, and heart failure, we added oral prednisolone (30 mg daily) to his prior anticoagulant regimen. The skin lesions and the pleural effusion improved rapidly, eventually disappearing without complication (fig. 4). On follow-up clinical examinations, no symptoms related to SLE or other collagen diseases were noted.

\section{Discussion}

The cause of the pleural effusion in this case continues to be unclear. Common causes of pleural effusion include malignancies, infectious diseases, pulmonary embolism, collagen vascular disease, and heart failure [3]. APS-related pleural effusion has rarely been reported, and those cases that have been reported appeared to be complications of accompanying pulmonary embolism, SLE, or catastrophic APS [4-6]. Pleuritis, which can induce pleural effusion, is the most common pleuropulmonary manifestation of SLE [7]. In the present case, after excluding these differential diagnoses, APS was determined to be the direct cause of the pleural effusion. However, a strong possibility still exists that the pleural effusion may be associated with occult collagen vascular disease, particularly SLE or lupus-like disease (LLD) heretofore undiagnosed. Several studies have suggested that patients with primary APS may 
develop SLE or LLD. A long-term follow-up study in 128 patients with primary APS demonstrated that 11 patients (8\%) developed SLE, while $6(5 \%)$ developed LLD during a median follow-up period of 8.2 years (range, 114 years) [8]. The results of this study suggest that the pleural effusion may be attributed to a coexisting condition like LLD, although our patient has not fulfilled American College of Rheumatology diagnostic SLE criteria to date. This may be supported by the fact that oral corticosteroid therapy was a remarkably effective treatment of the pleural effusion that had previously been unsuccessfully treated by anticoagulant therapy and repeated drainage.

Corticosteroids and immunosuppressants continue to be the treatment of choice for severe SLE complications, including pleural effusion [9]. Furthermore, the clinical manifestations of primary APS and APS associated with SLE are similar, which makes it more difficult to differentiate these diseases. As pleural effusion can be life-threatening, physicians should be aware of this entity. Corticosteroids might be an effective choice of treatment for intractable pleural effusion in APS patients.

\section{Disclosure Statement}

The authors declare no conflicts of interest.

\section{References}

$\checkmark 1$ Vianna JL, Khamashta MA, Ordi-Ros J, et al: Comparison of the primary and secondary antiphospholipid syndrome: a European multicenter study of 114 patients. Am J Med 1994;96:3-9.

2 Espinosa G, Cervera R, Font J, et al: The lung in the antiphospholipid syndrome. Ann Rheum 2002;61:195198.

-3 Heffner JE, Brown LK, Barbieri CA: Diagnostic value of tests that discriminate between exudative and transudative pleural effusions. Primary Study Investigators. Chest 1997;111:970-980.

4 Phatak S, Redkar N, Patil MA, Karnik ND: Antiphospholipid syndrome presenting as pulmonary thrombosis and pancreatitis in a young man. BMJ Case Rep 2012;2012:bcr2012006348.

5 Morita H, Daito H, Suwa T, et al: Overt congestive heart failure with mitral and aortic regurgitation due to antiphospholipid syndrome in a patient with systemic lupus erythematosus. Intern Med 2000;39:506-511.

6 Makhoul BF, Guralnik L, Azzam ZS: Catastrophic antiphospholipid syndrome presented with abdominal, pulmonary, and bone marrow complications. Rheumatol Int 2010;30:401-404.

7 Lynch DA: Lung disease related to collagen vascular disease. J Thorac Imaging 2009;24:299-309.

8 Gómez-Puerta JA, Martín H, Amigo MC, et al: Long-term follow-up in 128 patients with primary antiphospholipid syndrome: do they develop lupus? Medicine (Baltimore) 2005;84:225-230.

9 Rovenský J, Tuchynová A: Systemic lupus erythematosus in the elderly. Autoimmun Rev 2008;7:235-239. 


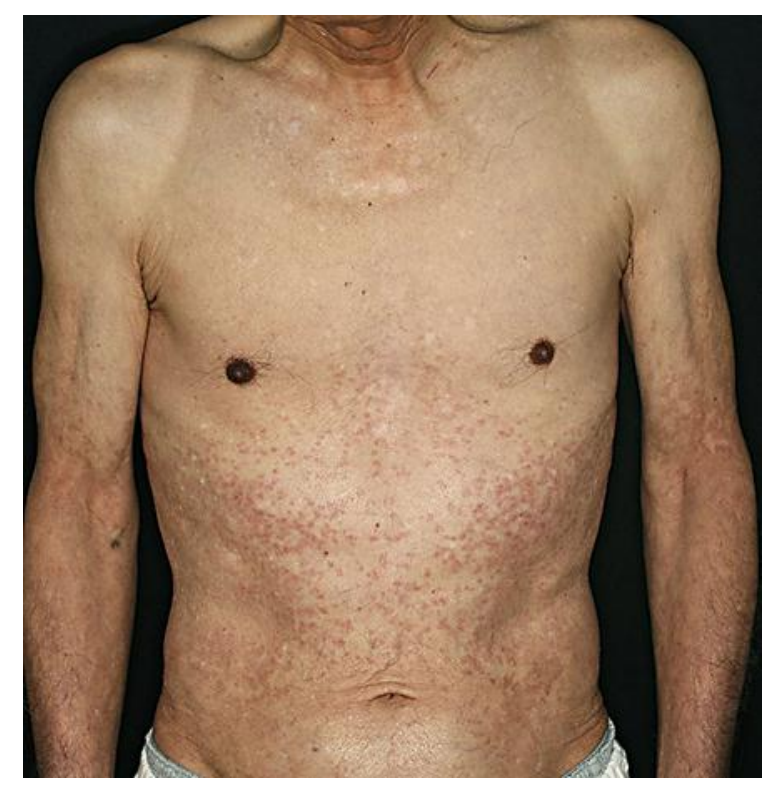

Fig. 1. Violaceous erythema, purpura, and pigmentation on the trunk and extremities.

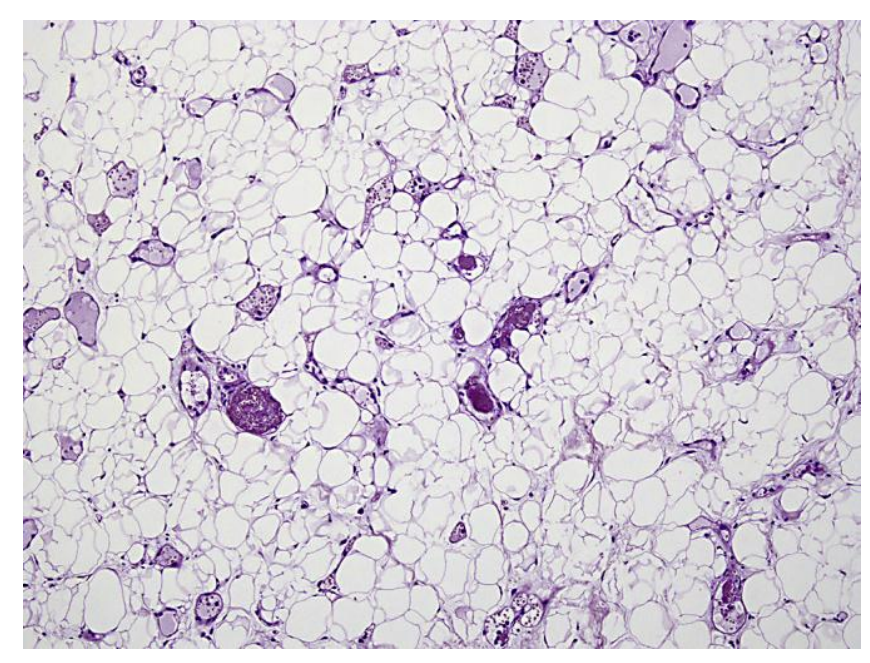

Fig. 2. Multiple fibrin thrombi in the subcutaneous vessels. HE. $\times 200$. 


\section{Case Reports in Dermatology}

Case Rep Dermatol 2013;5:198-202

DOI: $10.1159 / 000354135$

2013 S. Karger AG, Basel

www.karger.com/cde

Mitamura et al.: Antiphospholipid Syndrome Complicated by Unilateral Pleural Effusion

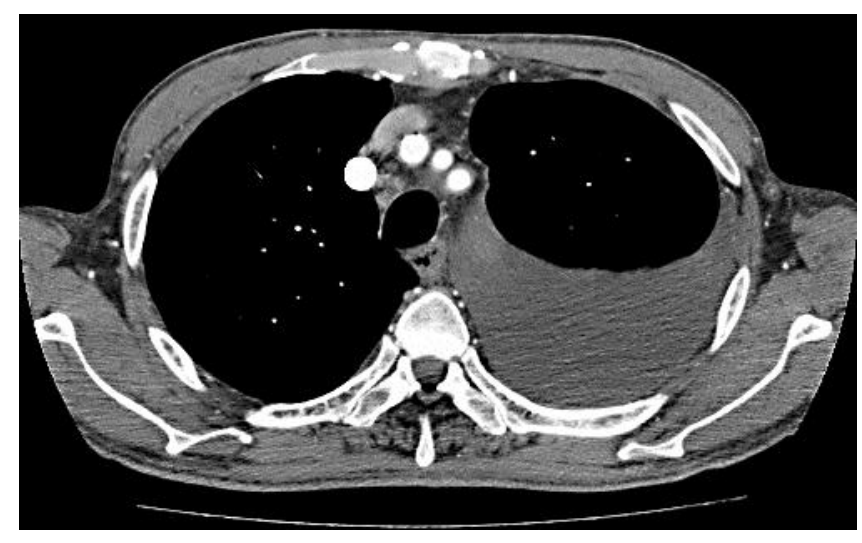

Fig. 3. Computed tomography demonstrates a large pleural effusion in the left lung.

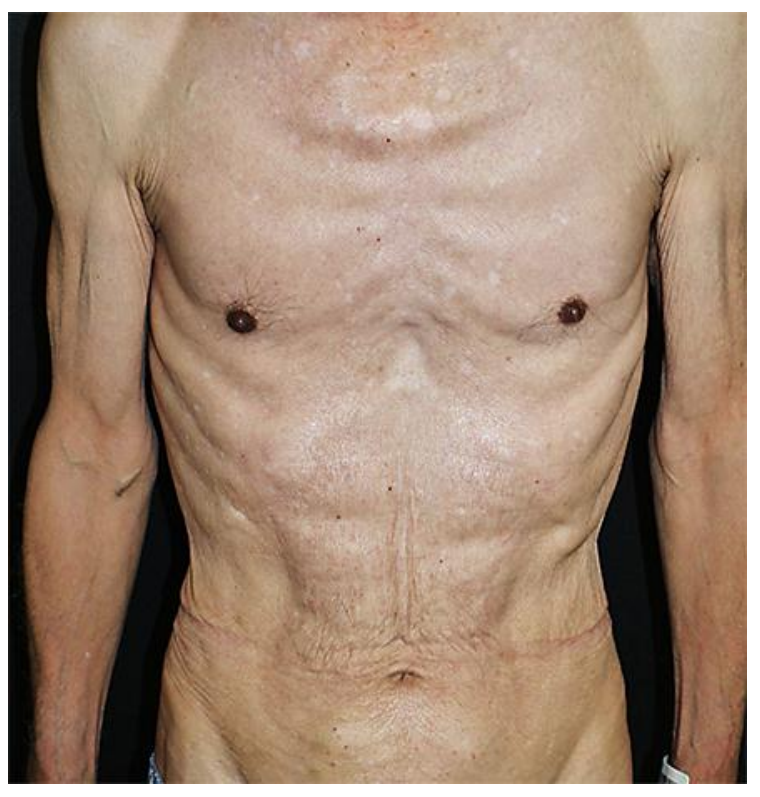

Fig. 4. After administration of oral prednisolone, the skin lesions improved rapidly. 